\title{
Nematicidal and Plant Growth-Promoting Activity of Enterobacter asburiae HK169: Genome Analysis Provides Insight into Its Biological Activities ${ }^{\mathbb{S}}$
}

\author{
Mira $\mathrm{Oh}^{1,2 \dagger}$, Jae Woo $\mathrm{Han}^{1 \dagger}$, Chanhui Lee ${ }^{3}$, Gyung Ja Choi ${ }^{1,2}$, and Hun Kim ${ }^{1,2 *}$ \\ ${ }^{1}$ Center for Eco-friendly New Materials, Korea Research Institute of Chemical Technology, Daejeon 34114, Republic of Korea \\ ${ }^{2}$ Department of Green Chemistry and Environmental Biotechnology, Korea University of Science and Technology, Daejeon 34113, Republic of Korea \\ ${ }^{3}$ Department of Plant and Environmental New Resources, Kyung Hee University, Yongin 17104, Republic of Korea
}

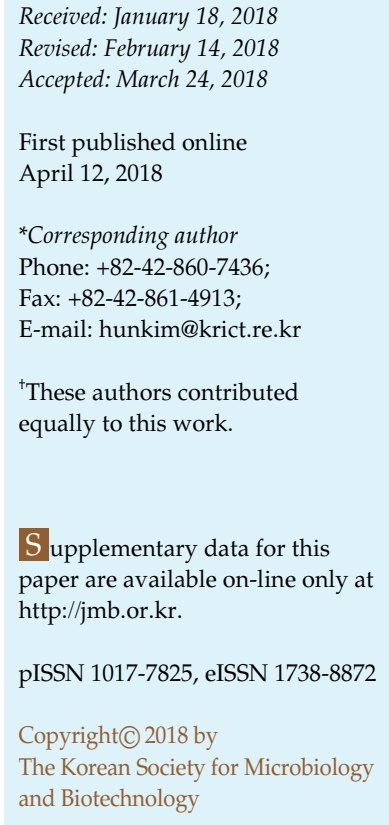

$S$ upplementary data for this paper are available on-line only at http://jmb.or.kr.

pISSN 1017-7825, eISSN 1738-8872

Copyright(C) 2018 by

The Korean Society for Microbiology and Biotechnology

\begin{abstract}
In the course of screening for microbes with nematicidal activity, we found that Enterobacter asburiae HK169 displayed promising nematicidal activity against the root-knot nematode Meloidogyne incognita, along with plant growth-promoting properties. Soil drenching of a culture of HK169 reduced gall formation by $66 \%$ while also increasing root and shoot weights by $251 \%$ and $160 \%$, respectively, compared with an untreated control. The cell-free culture filtrate of the HK169 culture killed all juveniles of M. incognita within $48 \mathrm{~h}$. In addition, the nematicidal activity of the culture filtrate was dramatically reduced by a protease inhibitor, suggesting that proteolytic enzymes contribute to the nematicidal activity of HK169. In order to obtain genomic information about the HK169 isolate related to its nematicidal and plant growth-promoting activities, we sequenced and analyzed the whole genome of the HK169 isolate, and the resulting information provided evidence that the HK169 isolate has nematicidal and plant growth-promoting activities. Taken together, these observations enable the future application of E. asburiae HK169 as a biocontrol agent for nematode control and promote our understanding of the beneficial interactions between E. asburiae HK169 and plants.
\end{abstract}

Keywords: Enterobacter asburiae, Meloidogyne incognita, biocontrol agent, plant growth promotion, genome analysis

\section{Introduction}

Root-knot nematodes (Meloidogyne spp.) are among the most destructive plant parasites and have a very wide host range [1]. An infection of root-knot nematodes causes the formation of galls on the root system, which interrupt the root uptake of water and nutrients [2]. Resistant cultivars, crop rotation, and chemical nematicides have been widely used to control root-knot nematodes. However, plant resistance against root-knot nematodes is often unstable or ineffective against certain species of root-knot nematodes [3]. Furthermore, the overuse of chemical nematicides has resulted in environmental pollution and has led to the emergence of resistant strains [4,5]. Therefore, instead of chemical control for root-knot nematodes, alternative strategies such as biological control agents are being explored [6].

A rhizosphere is a site where complex interactions among plant roots, soil, and soilborne microorganisms occur [6]. Plant parasitic nematodes and rhizobacteria occupy the same niche (rhizosphere), and some of these may have antagonistic or symbiotic relationships [6, 7]. Rhizobacteria can prevent the proliferation of nematodes by producing nematicidal substances such as antibiotics and cell-walldegrading enzymes or by the induction of systemic resistance in the host plants [8, 9]. Rhizobacteria also enhance the growth of plants by producing growthpromoting substances, such as indole-3-acetic acid (IAA), 1-aminocyclopropane-1-carboxylic acid (ACC) deaminase, and volatile compounds $[10,11]$. 
The Enterobacter species are gram-negative bacteria belonging to the family Enterobacteriaceae, and the genus Enterobacter has been one of the most rapidly expanding genera in the last decade [12]. Members of this genus are most commonly isolated from the environment, in particular from soil, plants, and fruits, but also from human sources [13]. Several strains of Enterobacter have been investigated as plant growth-promoting rhizobacteria that colonize plant roots and improve the health or yields of plants [14, 15]. Although plant growth-promoting rhizobacteria have been investigated widely as biological control agents for plant parasitic nematodes, the nematicidal nature of Enterobacter has been rarely described [16].

The present study was designed to examine the agricultural benefits of Enterobacter asburiae HK169 isolated from the root system of tomato plants. The activities of the HK169 isolate in both stimulating plant growth and controlling root-knot nematodes have been explored and discussed. In addition, a whole-genome analysis of E. asburiae HK169 enables the comprehensive identification and profiling of potential factors contributing to its beneficial role in plant growth-promoting and nematicidal activities.

\section{Materials and Methods}

\section{Antagonistic Bacterial Strain}

The rhizobacterial strain HK169 isolated from tomato roots has been deposited into the Korean Agricultural Culture Collection (KACC) with accession number KACC 81020BP. The isolated bacterial strain was maintained on tryptic soy agar or LuriaBertani (LB) agar medium for further study and was incubated at $30^{\circ} \mathrm{C}$ for 3 days to investigate its nematicidal activity.

\section{Nematode Eggs and Juveniles}

Root-knot nematode Meloidogyne incognita, identified previously by Hwang et al. [17], was proliferated on tomatoes (Lycopersicum esculentum Mill. cv. Seokwang; Seminis Vegetable Seeds, Korea) in a greenhouse at $28 \pm 5^{\circ} \mathrm{C}$. To obtain nematode eggs, tomato roots that had a severe case of galling due to an infection of M. incognita were submerged into a $1 \%$ sodium hypochlorite solution and then macerated with a blender. The ground plant materials were filtrated through a 45 and $25 \mu \mathrm{m}$ sieve, sequentially, after which the eggs were collected and rinsed with distilled water. To obtain second-stage juveniles, the collected eggs were allowed to hatch using modified Baermann funnels [18] at room temperature.

\section{In Vitro Mortality Bioassay}

$M$. incognita juveniles and eggs were used for a bioassay using 96-well tissue culture plates (SPL Life Sciences, Korea). Aliquots of $25 \mu \mathrm{l}$ suspensions containing approximately 50 juveniles and 150 eggs were placed in each well, after which the culture broth (or culture filtrate) of E. asburiae HK169 was added to the wells to reach final concentrations of $5 \%, 10 \%$, and $20 \%(\mathrm{v} / \mathrm{v})$. As a cell treatment, pelleted cells after centrifugation of the culture broth were resuspended in distilled water at $2 \times 10^{8}$ cells $/ \mathrm{ml}$ and then added to a juvenile solution to reach a final concentration of $10 \%$ (v/v). Abamectin ( $1 \mu \mathrm{g} / \mathrm{ml}$; Sigma-Aldrich, USA) and LB medium were used as a positive and negative control, respectively. After incubation for 48 or $72 \mathrm{~h}$, the juveniles were observed under a light microscope. Nematode juveniles were judged as dead if their bodies were straight and showed no movement. The rate of inhibition of nematode egg hatching was assessed under a light microscope at 7 days after treatment, and the egg hatch rate $(\mathrm{EH})$ was calculated as follows: $\mathrm{EH}=$ [number of juveniles/(number of eggs + number of juveniles) $] \times 100$. The experiment was conducted twice with three replicates. Mortality values were calculated according to Abbott's formula [19]: mortality $(\%)=[($ mortality percentage in treatment - mortality percentage in control $) /(100-$ mortality percentage in control)] $\times 100$. Additionally, hatch inhibition was calculated as follows: Hatch inhibition $(\%)=[(\mathrm{EH}$ in control $)-(\mathrm{EH}$ in treatment $) /(\mathrm{EH}$ in control) $] \times 100$.

To investigate if the protease of E. asburiae HK169 had an effect on the nematicidal activity, a protease-inhibitor cocktail (SigmaAldrich, USA) was added to the culture broth at a ratio of 1:9. The juvenile suspension ( 50 juveniles/well) was placed in each well, followed by the addition of $2.5 \%, 5 \%$, and $10 \%$ of the mixture containing the bacterial culture and the protease inhibitor. The nematicidal activity was evaluated $72 \mathrm{~h}$ after the treatment.

\section{In Vivo Assessment of Plant Disease Control}

To explore the degree of disease control efficacy, M. incognita eggs $(\sim 5,000)$ were inoculated into each pot containing a 3-weekold tomato seedling. After $1 \mathrm{~h}$, a 2-day-old culture $(20 \mathrm{ml})$ of E. asburiae HK169 was applied to each pot by drenching. A 2,000fold dilution of sunchungtan (active ingredient: 30\% fosthiazate; Farm Hannong, Korea) and a LB medium containing a $0.025 \%$ Tween 20 solution were used as positive and negative controls, respectively. After 6 weeks of incubation, the plants were carefully uprooted and washed free of sand particles. The galling index (GI) of the tomato roots was assessed in five phases depending on the formation level of galls $(0,0 \% ; 1,1-25 \% ; 2,26-$ $50 \% ; 3,51-75 \% ; 4,76-100 \%$ ) [20].

\section{Whole-Genome Sequencing and Genome Analysis}

The genomic DNA of E. asburiae HK169 was extracted using QIAamp genomic DNA kits (Qiagen, Germany), and the quantity and quality of the genomic DNA were determined using an Agilent 2100 Bioanalyzer (Agilent Technologies, USA). The 20-kb SMRTbell library was constructed and then sequenced with the PacBio RS II platform (Pacific Biosciences, USA). The resulting sequences were de novo assembled using the RS hierarchical genome assembly process 3.0 in the SMRT analysis program (ver. 2.3, Pacific Biosciences, USA). Genome annotation was predicted using Prokka ver. 1.11 and the prokaryotic genome annotation 
pipeline (PAGP ver. 3.3) of NCBI. For a whole-genome comparison of closely related strains, the average nucleotide identities were calculated with currently available genome sequences of Enterobacter spp. using OAT ver. 0.90 (http://www.ezbiocloud.net/tools/ orthoani). The gene clusters involved in the biosynthesis of secondary metabolites were analyzed with antiSMASH ver. 3.0 (http://antismash.secondarymetabolites.org).

\section{Phylogenetic Analysis}

To identify isolated bacterial strains, pairwise sequence similarities of the $16 \mathrm{~S}$ rRNA gene were determined with the most closely related strains, using a global alignment algorithm on the EzTaxon server (http://www.ezbiocloud.net/identify). The consensus DNA sequence of the gyrB gene was subjected to sequence-similarity searches using BLASTn (http://www.blastn. ncbi.nlm.nih.gov). The sequences of gyrB were aligned using ClustalW implemented in MEGA ver. 7, and distances were estimated according to the model of Tamura and Nei [21]. The robustness of the inferred trees was evaluated by a bootstrap analysis consisting of 1,000 resamplings, and the phylogenetic tree was generated using the neighbor-joining method.

\section{Statistical Analysis}

Data ( $n=3$ independent experiments) were subjected to a oneway ANOVA, and the means of the treatments were separated by Duncan's multiple range test using the R-software package (ver. 3.3.1). All values are expressed as the mean \pm standard deviation. Differences were considered statistically significant for $p$ values of less than 0.05 .

\section{Results and Discussion}

\section{Isolation and Identification of E. asburiae HK169}

In the course of screening for microbes with nematicidal activity, we found that rhizobacterial strain HK169 isolated from tomato roots showed strong nematicidal activities against the root-knot nematode $M$. incognita. This strain is gram-negative and rod-shaped with a cell size of 0.5-0.6 by $1.2-1.6 \mu \mathrm{m}$ (Figs. S1A and S1B). The colonies grown on LB agar media are creamy white, mucoid, translucent, and $5-9 \mathrm{~mm}$ in diameter after $48 \mathrm{~h}$ of incubation at $30^{\circ} \mathrm{C}$ (Fig. S1).

Sequence alignment of the $16 \mathrm{~S}$ rRNA gene revealed that the HK169 strain belongs to the genus Enterobacter and is most closely related to Enterobacter cloacae MNCRE16 (JYMH01000051), Enterobacter tabaci YIM Hb-3 ${ }^{\mathrm{T}}$ (KP990658), Enterobacter asburiae $\mathrm{JCM} 6051^{\mathrm{T}}$, and Enterobacter cancerogenus LMG 2693 ${ }^{\mathrm{T}}$ (Z96078), with 99.5\%, 99.3\%, 99.2\%, and 99.0\% similarity, respectively. A phylogenetic analysis based on gyrB gene sequences showed that the HK169 strain was the most close to E. asburiae strains (Fig. S1C). The HK169 strain shared very high average nucleotide identity values (>97\%) with E. asburiae strains E20 (CP012999), CAV 1043 (CP011591), L1 (CP007546), and ATCC 35953 (CP011863). Thus, the HK169 strain was identified as E. asburiae.

\section{In Vitro Nematicidal Activity of E. asburiae HK169}

To investigate the nematicidal activity of $E$. asburiae HK169, an E. asburiae HK169 culture broth, cell-free filtrate, and cells were individually treated to nematode juveniles and eggs in vitro. The treatment with $10 \%$ culture broth (v/v) resulted in $98 \%$ juvenile mortality at $48 \mathrm{~h}$ after treatment and suppressed egg hatching by $54 \%$ at 7 days after treatment, rates that were comparable to those of the abamectin treatment (Table 1); the juvenile mortality and egg hatch suppression were increased depending on the concentration of the treatment. Furthermore, we observed that the nematode cuticle was thinner than those of the non-treatment control group at $72 \mathrm{~h}$ after the treatment (data not shown). When the culture filtrate of E. asburiae HK169 was exposed to juveniles, we observed that all juveniles were dead in $48 \mathrm{~h}$ (Fig. 1A). However, when E. asburiae HK169 cells were utilized, the mortality rate was less than $3 \%$ at $48 \mathrm{~h}$ after the treatment, although the mortality rate increased, reaching $49 \%$ at $72 \mathrm{~h}$ (Fig. 1A). Thus, our results showed that nematicidal substances may be released from the cells and may accumulate in the medium.

To identify the nematicidal compounds from the culture filtrate of E. asburiae HK169, the culture filtrate was partitioned with the organic solvents ethyl acetate and butanol. Unexpectedly, most of the nematicidal activity disappeared in all of the organic solvent fractions, through an aqueous layer remained after partitioning (data not

Table 1. In vitro nematicidal activity of the Enterobacter asburiae HK169 culture broth ${ }^{\mathrm{a}}$.

\begin{tabular}{lccc}
\hline Treatment & Concentration & $\begin{array}{c}\text { Mortality (\%) } \\
\text { of J2s }\end{array}$ & $\begin{array}{c}\text { Hatching } \\
\text { inhibition }(\%) \\
\text { of eggs }\end{array}$ \\
\hline HK169 & $10 \%$ & $98.2 \pm 2.4 \mathrm{z}^{\mathrm{b}}$ & $53.7 \pm 5.7 \mathrm{z}$ \\
& $5 \%$ & $70.5 \pm 10.5 \mathrm{y}$ & $40.6 \pm 0 \mathrm{y}$ \\
Abamectin & $1 \mu \mathrm{g} / \mathrm{ml}$ & $97.6 \pm 3.4 \mathrm{z}$ & $55.6 \pm 4.7 \mathrm{z}$ \\
\hline
\end{tabular}

${ }^{\text {a Mortality and egg hatching inhibition of M. incognita were investigated at } 48 \mathrm{~h}}$ and 7 days after treatment, respectively.

${ }^{b}$ Each value represents the mean \pm standard deviation of three replications. Different small letters in each column indicate a significant difference at $p<0.05$ (Duncan's test). 
A

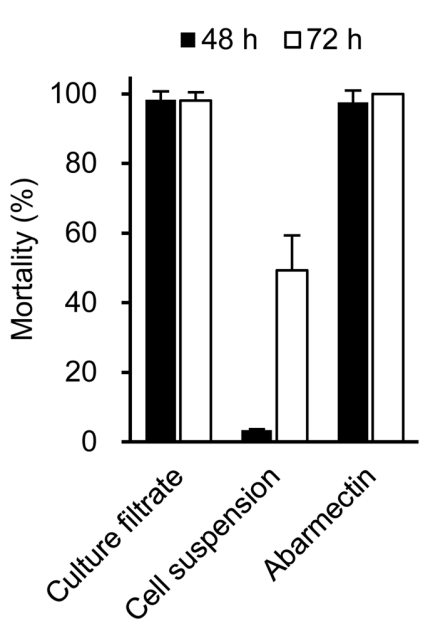

B

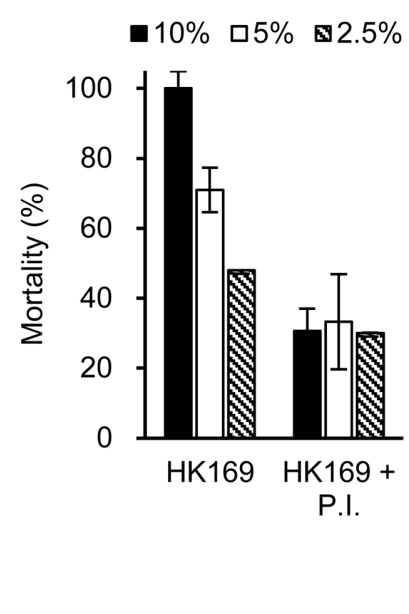

Fig. 1. In vitro nematicidal effects of Enterobacter asburiae HK169 on second-stage juveniles of Meloidogyne incognita.

(A) Nematicidal activity of cell-free culture filtrate and cell suspension of the HK169 strain. (B) Effects of a protease-inhibitor cocktail on the nematicidal activity of the culture filtrate of the HK169 strain.

shown). Although the ethyl acetate fraction showed low nematicidal activity $(20 \%)$, a possible active compound in the ethyl acetate fraction is likely to be responsible for a small portion of the nematicidal activity of the HK160 isolate, as the ethyl acetate fraction showed nematicidal activity at high concentrations exceeding 2,500 $\mu \mathrm{g} / \mathrm{ml}$ (data not shown).

Extracellular enzymes, including serine proteases, chitinases, and collagenases, have been described as important factors that can degrade the structural constituents of nematode surfaces or eggshells [22]. Extracellular proteases have been intensively studied in relation to antagonistic fungi and bacteria against pathogenic nematodes [23]. In particular, a number of nematicidal proteases have been identified from Bacillus spp. [24, 25]. In this study, to determine whether proteases of the HK169 strain have nematicidal activity, bacterial cultures were exposed to juveniles with a protease-inhibitor cocktail. Treatments of $10 \%, 5 \%$, and $2.5 \%$ of the bacterial culture without the protease-inhibitor cocktail showed $100 \%, 71 \%$, and $48 \%$ mortality rates, respectively, with the mortality rate depending on the culture concentration. In contrast, bacterial cultures containing the protease-inhibitor cocktail showed dramatically reduced mortalities compared with the treatment without the protease-inhibitor cocktail, suggesting that the presence of extracellular proteases is associated with the nematicidal activity of E. asburiae HK169 (Fig. 1B). Unexpectedly, we

A

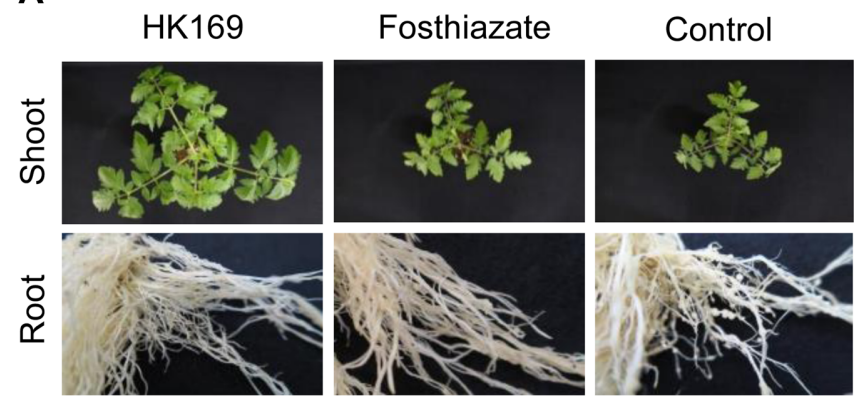

B
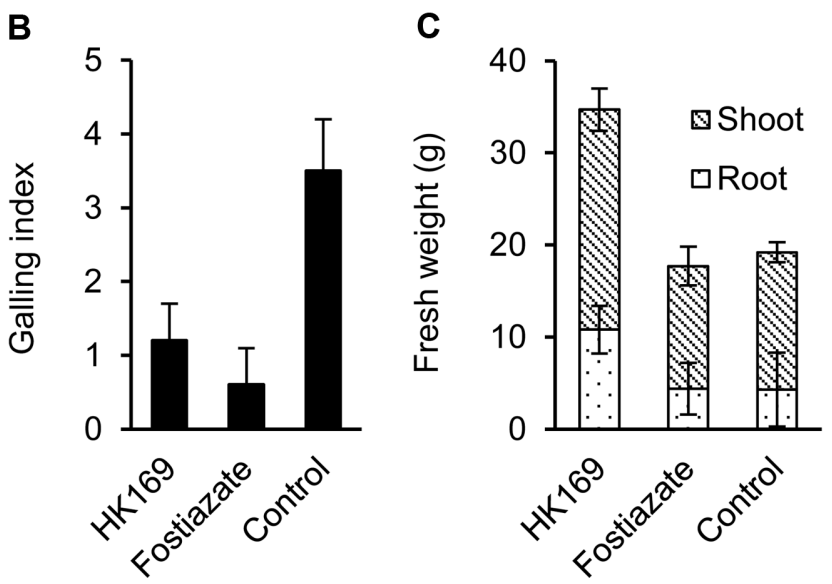

Fig. 2. Nematicidal and plant growth-promoting activities of Enterobacter asburiae HK169.

(A) Root gall formation and shoot growth of tomato plants treated with the culture broth of HK169 or the nematicide fosthiazate. (B) In vivo nematicidal activity of the HK169 strain on tomato plants infected with Meloidogyne incognita, which was based on the gall formation. (C) Plant growth-promoting effect of the HK169 strain.

observed that the addition of the protease-inhibitor cocktail led to nearly $30 \%$ mortality regardless of the culture concentration (Fig. 1B). For this phenomenon, we cannot exclude the possibility that E. asburiae HK169 may possess other nematicidal mechanisms beyond proteases or that proteinase inhibitors may be toxic to nematodes and insects [26], although we did not observe nematicidal toxicity of the proteinase-inhibitor cocktail in this study.

\section{In Vivo Nematicidal Activity and Plant Growth Promotion} by E. asburiae HK169

The culture broth of E. asburiae HK169 exhibited a reduction in root-knot disease caused by the infection of M. incognita on tomato roots (Figs. 2A and 2B). Tomato plants treated with the HK169 culture broth showed 65\% less root gall formation compared with the non-treatment 
control (Fig. 2B). Furthermore, treatment with the HK169 culture broth stimulated the growth of tomato plants (Figs. 2A and 2C). The means of the root and shoot weights of the tomato seedlings treated with the HK169 culture broth were increased by $251 \%$ and $160 \%$ relative to the corresponding control values, respectively (Fig. 2C). As a control, when the commercial nematicide fosthiazate was applied to the tomato plants, the galling index was significantly reduced compared with that of the nontreatment control, whereas the plant growth outcome did not differ from that of the control plants (Fig. 2).

Duponnois et al. [27] described the potential of E. cloacae to stimulate plant growth and to reduce the egg formation and hatching of the root-knot nematode $M$. incognita. The possible mechanism was proposed to be Enterobacter spp. improving the levels of nitrogen fixation, IAA production, and ACC deamination of the plants [10, 13, 28]. Some plant growth-promoting rhizobacteria interfere with the relationship between plant-parasitic nematodes and their hosts, through which rhizobacteria produce metabolites that reduce egg hatching, nematode attraction, and degradation of specific root exudates controlling nematode behavior [29]. However, less is known about the possible mechanisms of action with regard to controlling plantparasitic nematodes by Enterobacter species.

\section{Genome Analysis of E. asburiae HK169}

In order to obtain genomic information about E. asburiae HK169 related to its plant growth-promoting and nematicidal activities, the genome sequence of the HK169 isolate was analyzed. A total of 1,372,285,639 nucleotides based on 177,762 reads (N50 size 10,718 and mean subread length $7,719)$ with 221-fold coverage of the genome were generated, and the genome sequence was deposited into the GenBank database with accession number CP017087. The wholegenome sequence of HK169 features a single, circular chromosome of 4,551,186 bp in length, with $56.2 \% \mathrm{G}+\mathrm{C}$ content and with no plasmid detected. In total, 4,141 coding DNA regions were identified with 83 tRNA, 25 rRNA, 12 ncRNA, and 45 pseudo genes.

Based on RAST functional categories (http:/ / rast.nmpdr. org), the carbohydrate metabolism subsystem (14.8\%) ranks as the largest, followed by amino acid metabolism $(12.4 \%)$ and protein metabolism $(9.0 \%)$ (Fig. 3A). The subsystem of protein metabolism includes 43 proteindegradation-related open reading frames, including the putative TldE-TldD proteolytic complex, protein-degrading enzymes, aminopeptidases (E.C. 3.4.11), metallocarboxypeptidases (E.C. 3.4.17), dipeptidases (E.C. 3.4.13), bacterial
A

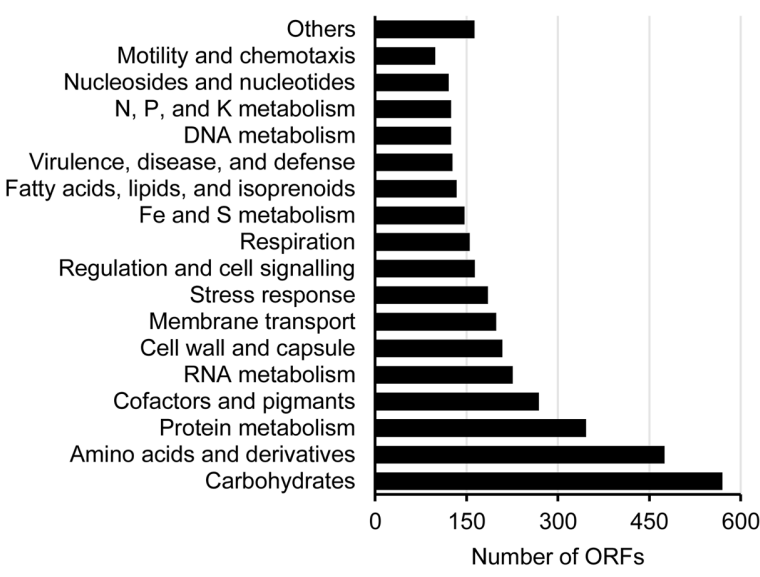

B

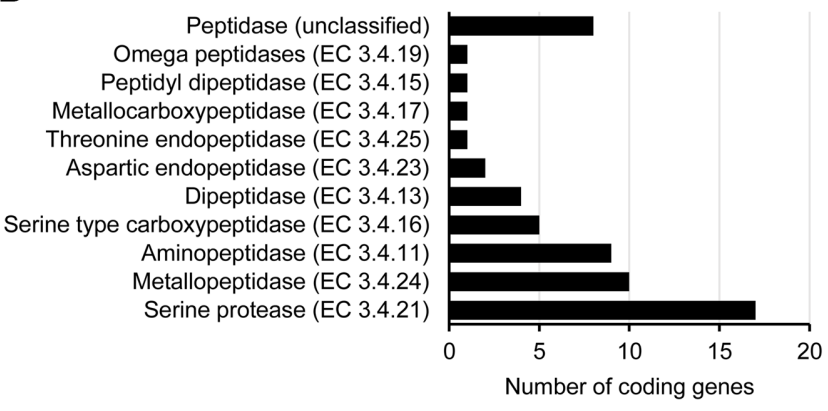

Fig. 3. Subsystem category distribution of major protein coding genes of Enterobacter asburiae HK169 as annotated by the RAST annotation server (A) and 59 putative genes encoding proteolytic enzymes in the HK169 genome (B).

proteasome, omega peptidases (E.C. 3.4.19), and bacterialATP-dependent proteolytic enzymes (Fig. 3B). Virulence factors such as toxins were not detected in the subcategories of virulence, disease, and defense.

\section{Protease-Related Genes in E. asburiae HK169}

The genome of the HK169 isolate contains 59 proteaserelated genes, including 17 serine proteases and 10 metallopeptidases (Fig. 3B). Several serine proteases have been proven to be involved in the antagonistic action of bacteria against plant parasitic nematodes, in which protein components hydrolyze the eggshell, cuticle, and intestine $[24,30]$. The bacterial strain Pseudomonas fluorescens CHA0 produces extracellular serine protease AprA for its nematicidal activity [31]. The deficient mutant of the aprA gene or its regulatory gacA gene shows lost or impaired nematicidal activity compared with the wild type [31]. Paiva et al. [23] evaluated the nematicidal activities of 47 bacterial strains against the pinewood nematode and 
identified two nematicidal proteins consisting of a subtilisintype serine protease and a serralysin-like metalloprotease from Serratia marcescens A88copa13. Recently, the nematicidal metalloproteinases Bmp1 and ColB were identified from Bacillus thuringiensis [25, 32]. These examples support our hypothesis that proteolytic enzymes encoded in the genome of HK169 contribute to its nematicidal activity.

\section{Secondary Metabolites of E. asburiae HK169}

In the complete genome of the HK169 isolate, we found several gene clusters for secondary metabolites, such as siderophore and aryl polyene (Table 2). Biosynthetic gene loci for two siderophores, aerobactin (EnteroDNA1_0122801236) and enterobactin (EnteroDNA1_03893-03912), were present in this genome. Bacteria have developed several distinct mechanisms by which to compete for iron, an element whose availability often limits microbial growth. Costa and Loper [8] reported that aerobactin and enterobactin are required for iron acquisition by E. cloacae. A mutant strain of E. cloacae deficient in the production of both aerobactin and enterobactin failed to grow on an ironlimited medium. Siderophore-mediated iron deficiency in the rhizosphere is often proposed as a mechanism of action in the reduction of soilborne pathogens [33]. The presence of an efficient iron uptake system can therefore contribute to protect the host plant against pathogenic nematode infections. In addition, the aryl polyene biosynthetic gene cluster (EnteroDNA1_02481-02499) was found in the HK169 genome. Both symbiotic and pathogenic bacteria of eukaryotic cells are likely to encounter oxidative stress frequently from their hosts during colonization or infection. Membrane-bound aryl polyene protects gram-negative bacterial cells from oxidative damage by reducing the concentrations of free radicals [34]. Many bacteria that interact with higher plants or animals harbor the aryl polyene biosynthetic gene cluster [35].

Table 2. Gene clusters found in the genome of Enterobacter asburiae HK169, which are related to the biosynthesis of secondary metabolites.

\begin{tabular}{lll}
\hline \multicolumn{1}{c}{ Gene cluster type ${ }^{\mathrm{a}}$} & Gene locus $^{\mathrm{b}}$ & $\begin{array}{c}\text { Most similar known } \\
\text { gene cluster }\end{array}$ \\
\hline Hserlactone & $00630-00631$ & LuxI/R quorum sensing \\
Siderophore & $01228-01236$ & Aerobactin \\
Aryl polyene & $02481-02499$ & APE $_{E c}$ \\
Non-ribosomal peptide & $03893-03912$ & Enterobactin \\
\hline
\end{tabular}

${ }^{\text {a }}$ Predicted by antiSMASH 3.0.

${ }^{b}$ Designated by locus tag numbers (the EnteroDNA1_prefix being deleted for clarity) that are based on HK169 genome annotations.

\section{Genome Analysis of E. asburiae HK169 in Terms of Plant Growth Promotion}

We also found that several key genes are involved in plant growth-promoting activities, such as in the production of plant growth-regulating substances, biofilm formation, and the production of volatile compounds. Endophytic and rhizosphere bacteria enhance plant growth through the synthesis of the auxin-class plant hormone IAA. Although the production of IAA by the HK169 isolate was not experimentally demonstrated in this study, the tryptophan degradation pathway would be capable of producing indole-3-pyruvate, through which the aromatic amino acid aminotransferase (EnteroDNA1_03084) catalyzes the transamination of L-tryptophan. The indole-3-pyruvate decarboxylase IpdC (EnteroDNA1_01540) converts indole3-pyruvate into indole-3-acetaldehyde. Indole-3-acetaldehyde can also be synthesized from tryptamine, a decarboxylated product of L-tryptophan, by monoamine oxidase (EnteroDNA1_00463). The indole-3-acetaldehyde is further oxidized to IAA by indole-3-acetaldehyde dehydrogenase (EnteroDNA1_02926). Indole-3-acetylasparate hydrolase (EnteroDNA1_00376) is important in the regulation of indole-3-acetic acid metabolism in plants.

The $\mathrm{N}$-acyl homoserine lactone is a signal molecule in bacterial quorum sensing that serves as a means of communication between bacteria [36]. The acyl homoserine lactone synthase (luxI, EnteroDNA1_00630) and its transcriptional activator ( $u x R$, EnteroDNA1_00631) were found in the genome of HK169 (Table 2). The quorumsensing system mediated by LuxI/LuxR-like proteins has been described as responsible for biofilm formation [37], and in this study, an early stage of biofilm formation of the HK169 isolate was observed by scanning electron microscopy (Fig. S1). In addition to biofilm formation, the quorumsensing system mediated by LuxI/LuxR-like proteins is known to be involved in bacterial root colonization, which is an important factor in plant growth promotion by rhizobacteria and for the biocontrol activity of rootassociated Pseudomonas fluorescens 2P24 [37].

Volatile compounds such as acetoin and 2,3-butanediol are emitted by some plant growth-promoting rhizobacteria to enhance plant growth [11]. The main pathway for the production of acetoin and 2,3-butanediol by HK169 is via the budABC operon. Acetolactate synthase (budB, EnteroDNA1_03878) converts pyruvate to acetolactate, which is subsequently converted by $\alpha$-acetolactate decarboxylase (budA, EnteroDNA1_03877) into acetoin. Acetoin is released by bacteria or is subsequently converted into 2,3-butanediol by acetoin reductase (budC, EnteroDNA1_03879). Under 
aerobic condition, acetolactate is spontaneously converted into diacetyl (2,3-butanedione), which in turn can be converted into acetoin by diacetyl reductase (butA, EnteroDNA1_00699).

In terms of nitrogen fixation for plant growth, unlike other nitrogen-fixing Enterobacter strains, HK169 lacks the nif genes required for nitrogen fixation. However, the HK169 genome contains the genes required for the nitrate reduction pathways. Nitrate transport and nitrate/nitrite reduction genes are present within two operons (narIJHGKXL and nasAD-cmpCB-nrtA nasR, EnteroDNA1_00946-00959) that can be separated by a putative invasin gene. Other regions involved in nitrite reduction (nasD and nirD, EnteroDNA1_02428-02429) and an ammonium uptake transporter (amtB, EnteroDNA1_03753) and its regulator (EnteroDNA1_03752), as well as a nitrate/nitrite sensor protein (narX, EnteroDNA1_01583), were also found on the HK169 genome.

In summary, we have isolated and identified E. asburiae HK169 from tomato roots, showing nematicidal activity against $M$. incognita and plant growth-promoting properties. In addition, our observations that the nematicidal activity of the culture filtrate was dramatically reduced by a protease inhibitor suggested that proteolytic enzymes contribute to the nematicidal activity of HK169. Based on a whole-genome analysis of the HK169 isolate, our study provides evidence that the HK169 isolate is capable of nematicidal and plant growth-promoting activities. Taken together, our results will contribute to the application of improved microbial agents in agriculture.

\section{Acknowledgments}

This work was supported by the Next-Generation BioGreen21 Program (No. PJ01180201) of the Rural Development Administration and is part of the SKO1707C12 project of the Korea Research Institute of Chemical Technology, Republic of Korea.

\section{Conflict of Interest}

The authors have no financial conflicts of interest to declare.

\section{References}

1. Sasser JN. 1977. Worldwide dissemination and importance of the root-knot nematodes, Meloidogyne spp. J. Nematol. 9: 26-29.
2. Bird AF. 1974. Plant response to root-knot nematode. Annu. Rev. Phytopathol. 12: 69-85.

3. Brown CR, Mojtahedi H, Santo GS, Williamson VM. 1997. Effect of the $M i$ gene in tomato on reproductive factors of Meloidogyne chitwoodi and M. hapla. J. Nematol. 29: 416-419.

4. Tom T, David R. 1986. In vitro testing for nonfumigant nematicide resistance in Meloidogyne incognita and Pratylenchus vulnus. Rev. Nématol. 9: 385-390.

5. Vu TT, Kim H, Tran VK, Dang QL, Nguyen HT, Kim H, et al. 2016. In vitro antibacterial activity of selected medicinal plants traditionally used in Vietnam against human pathogenic bacteria. BMC Complement. Altern. Med. 16: 32-37.

6. Kerry BR. 2000. Rhizosphere interactions and the exploitation of microbial agents for the biological control of plantparasitic nematodes. Annu. Rev Phytopathol. 38: 423-441.

7. Vessey JK. 2003. Plant growth promoting rhizobacteria as biofertilizers. Plant Soil 255: 571-586.

8. Costa JM, Loper JE. 1994. Characterization of siderophore production by the biological control agent Enterobacter cloacae. Mol. Plant Microbe Interact. 7: 440-448.

9. Dahiya N, Tewari R, Tiwari RP, Hoondal GS. 2005. Production of an antifungal chitinase from Enterobacter sp. NRG4 and its application in protoplast production. World J. Microbiol. Biotechnol. 21: 1611-1616.

10. Li J, Daniel H, Charles TC, Glick BR. 2000. An ACC deaminase minus mutant of Enterobacter cloacae UW4 no longer promotes root elongation. Curr. Microbiol. 41: 101-105.

11. Ryu CM, Farag MA, Hu CH, Reddy MS, Wei HX, Paré PW, et al. 2003. Bacterial volatiles promote growth in Arabidopsis. Proc. Nat. Acad. Sci. USA 100: 4927-4932.

12. Abbott SL. 2011. Klebsiella, Enterobacter, Citrobacter, Serratia, Plesiomonas, and other Enterobacteriaceae, pp. 639-657. In Versalovic J, Carroll K, Funke G, Jorgensen J, Landry M, Warnock D (eds.), Manual of Clinical Microbiology, 10th Ed. American Society of Microbiology, Washington, DC.

13. Peng G, Zhang W, Luo H, Xie H, Lai W, Tan Z. 2009. Enterobacter oryzae sp. nov., a nitrogen-fixing bacterium isolated from the wild rice species Oryza latifolia. Int. J. Syst. Evol. Microbiol. 59: 1650-1655.

14. Saleh SS, Glick BR. 2001. Involvement of gacS and rpoS in enhancement of the plant growth-promoting capabilities of Enterobacter cloacae CAL2 and UW4. Can. J. Microbiol. 47: 698-705.

15. Shoebitz M, Ribaudo CM, Pardo MA, Cantore ML, Ciampi L, Curá JA. 2009. Plant growth promoting properties of a strain of Enterobacter ludwigii isolated from Lolium perenne rhizosphere. Soil Biol. Biochem. 41: 1768-1774.

16. Siddiqui ZA, Futai K. 2009. Biocontrol of Meloidogyne incognita on tomato using antagonistic fungi, plant-growth-promoting rhizobacteria and cattle manure. Pest Manag. Sci. 65: 943-948.

17. Hwang SM, Park MS, Kim JC, Jang KS, Choi YH, Choi GJ. 2014. Occurrence of Meloidogyne incognita infecting resistant cultivars and development of an efficient screening method 
for resistant tomato to the $M i$-virulent nematode. Korean J. Hort. Sci. Technol. 32: 217-226.

18. Hooper DJ. 1986. Culturing nematodes and related experimental techniques, pp. 133-157. In Southey JF (ed.), Laboratory Methods for Work with Plant and Soil Nematodes. H. M. Stationery Office, London, UK.

19. Abbott WS. 1925. A method of computing the effectiveness of an insecticide. J. Econ. Entomol. 18: 265-267.

20. Jang JY, Choi YH, Shin TS, Kim TH, Shin K-S, Park HW, et al. 2016. Biological control of Meloidogyne incognita by Aspergillus niger F22 producing oxalic acid. PLoS One 11: e0156230.

21. Tamura K, Nei M. 1993. Estimation of the number of nucleotide substitutions in the control region of mitochondrial DNA in humans and chimpanzees. Mol. Biol. Evol. 10: 512-526.

22. Castaneda-Alvarez C, Prodan S, Rosales IM, Aballay E. 2016. Exoenzymes and metabolites related to the nematicidal effect of rhizobacteria on Xiphinema index Thorne \& Allen. J. Appl. Microbiol. 120: 413-424.

23. Paiva G, Proença DN, Francisco R, Verissimo P, Santos SS, Fonseca L, et al. 2013. Nematicidal bacteria associated to pinewood nematode produce extracellular proteases. PLoS One 8: e79705.

24. Huang X, Tian B, Niu Q, Yang J, Zhang L, Zhang K. 2005. An extracellular protease from Brevibacillus laterosporus G4 without parasporal crystals can serve as a pathogenic factor in infection of nematodes. Res. Microbiol. 156: 719-727.

25. Luo X, Chen L, Huang Q, Zheng J, Zhou W, Peng D, et al. 2013. Bacillus thuringiensis metalloproteinase Bmp1 functions as a nematicidal virulence factor. Appl. Environ. Microbiol. 79: 460-468.

26. Haq SK, Atif SM, Khan RH. 2004. Protein proteinase inhibitor genes in combat against insects, pests, and pathogens: natural and engineered phytoprotection. Arch. Biochem. Biophys. 431: 145-159.

27. Duponnois R, Amadou M, Mateille T. 1999. Beneficial effects of Enterobacter cloacae and Pseudomonas mendocina for biocontrol of Meloidogyne incognita with the endosporeforming bacterium Pasteuria penetrans. Nematology 1: 95-101.

28. Kim KY, Jordan D, McDonald GA. 1997. Effect of phosphate- solubilizing bacteria and vesicular-arbuscular mycorrhizae on tomato growth and soil microbial activity. Biol. Fertil. Soils 26: 79-87.

29. Sikora RA, Hoffmann-Hergarten S. 1993. Biological control of plant parasitic nematodes with plant-health-promoting rhizobacteria, pp. 166-172. In Lumsden RD, Vaughn JL (eds.), Pest Management: Biotechnology Based Technologies. American Chemical Society, Washington, DC.

30. Niu Q, Huang X, Zhang L, Xu J, Yang D, Wei K, et al. 2010. A Trojan horse mechanism of bacterial pathogenesis against nematodes. Proc. Nat. Acad. Sci. USA 107: 16631-16636.

31. Siddiqui IA, Haas D, Heeb S. 2005. Extracellular protease of Pseudomonas fluorescens CHA0, a biocontrol factor with activity against the root-knot nematode Meloidogyne incognita. Appl. Environ. Microbiol. 71: 5646-5649

32. Peng D, Lin J, Huang Q, Zheng W, Liu G, Zheng J, et al. 2016. A novel metalloproteinase virulence factor is involved in Bacillus thuringiensis pathogenesis in nematodes and insects. Environ. Microbiol. 18: 846-862.

33. Oostendorp M, Sikora RA. 1989. Seed treatment with antagonistic rhizobacteria for the suppression of Heterodera schachtii early root infection of sugar beet. Rev. Nematol. 12: 77-83.

34. Schöner TA, Gassel S, Osawa A, Tobias NJ, Okuno Y, Sakakibara Y, et al. 2016. Aryl polyenes, a highly abundant class of bacterial natural products, are functionally related to antioxidative carotenoids. Chembiochem 17: 247-253

35. Cimermancic P, Medema MH, Claesen J, Kurita K, Brown LCW, Mavrommatis K, et al. 2014. Insights into secondary metabolism from a global analysis of prokaryotic biosynthetic gene clusters. Cell 158: 412-421.

36. Schaefer AL, Val DL, Hanzelka BL, Cronan JE, Greenberg EP. 1996. Generation of cell-to-cell signals in quorum sensing: acyl homoserine lactone synthase activity of a purified Vibrio fischeri LuxI protein. Proc. Nat. Acad. Sci. USA 93: 9505-9509.

37. Wei HL, Zhang LQ. 2006. Quorum-sensing system influences root colonization and biological control ability in Pseudomonas fluorescens 2P24. Antonie Van Leeuwenhoek 89: 267-280. 